\section{Disease risks derived from genetic variants need clinical context}

To the the Editor:

he recent article by Mihaescu et al. ${ }^{1}$ makes important points regarding the impact of updates to risk factors and the limitations of disease risk estimates derived from genetic variants during a time of active discovery. However, there is a fundamental assumption made, both in this work and in the presentations of disease risk from commercial companies offering genome-wide scans, that is worthy of challenge. The threshold used is the population average. By presenting risks in relationship to the average in the overall population, clinical utility of the population average is implied, though this assumption most often is not supported.

In the Rotterdam Study population used by Mihaescu et al., the average risk of type 2 diabetes, calculated using both incident and prevalent cases, is reported as $20 \%$. Based on the single $T C F 7 L 2$ variant, predicted risks were $17.6 \%, 20.8 \%$, and $28.0 \%$ in the CC, CT, and TT genotype groups, respectively. So, consistent with the risk reporting used by direct-to-consumer companies offering full genome scans, risk of type 2 diabetes is deemed "below average" for CC individuals and "above average" for CT and TT individuals. However, what is the meaning of "above average" risk, particularly for the CT individuals, who make up about $40 \%$ of the population, ${ }^{2}$ and in whom the predicted risk is $20.8 \%$ ? In addition, what are the implications of comparing the risk in one subgroup of a population to the risk in the full population, when variants are common and subgroups make up a substantial portion of the total population (in this case $40 \%$ )?

Some well-studied, clinically developed risk scores have corresponding thresholds used in clinical care. The Framingham risk score, for example, provides estimates of the 10-year risk of heart attack or dying from coronary heart disease, based on a patient's age, gender, smoking status, diabetes status, blood pressure, and cholesterol. ${ }^{3}$ Current guidelines for prescribing cholesterol lowering therapy from the National Cholesterol Education Program-(ACT III) incorporate ranges of Framingham 10 -year risk $(<10 \%, 10-20 \%$, and $>20 \%),{ }^{4}$ and physicians may use the threshold of $20 \%$ when making treatment decisions. Hence, in this setting, a threshold of $20 \%$ for Framingham 10 -year risk has utility, and classifications and reclassifications based on this cutoff are consequential.

Returning to the example of type 2 diabetes, one might attempt to put the risks of disease based on genetic variants within clinical context by referring to comparable risk estimates in the clinical literature. The relationship between body mass index (BMI) and risk of diabetes has been established. Also, Narayan et al. ${ }^{5}$ estimated the remaining life-time risk of type 2 diabetes at age 18 years to be $19.8 \%$ for men of average weight $\left(18.5 \leq \mathrm{BMI}<25 \mathrm{~kg} / \mathrm{m}^{2}\right), 29.7 \%$ for overweight men $(25 \leq$ BMI $\left.<30 \mathrm{~kg} / \mathrm{m}^{2}\right)$, and $57.0 \%$ for obese men $(30 \leq$ BMI $<35$ $\mathrm{kg} / \mathrm{m}^{2}$ ). Similar estimates of remaining life-time risk of type 2 diabetes at age 18 years in women were $17.1 \%, 35.4 \%$, and $54.6 \%$, respectively. Based on these estimates, remaining lifetime risks at age 18 years of $30-35 \%$ (overweight) and greater (55-57\%, obese) provide a clinical framework, and possible thresholds, for reported risks. (To apply these cutoffs to the predicted risks presented by Mihaescu et al., however, one would have to demonstrate that the predicted risks, which might be interpreted as life-time risks of developing disease from birth to an average age of 69.5 years, are comparable with remaining life-time risk at age 18 years.)
Another approach to presenting risk estimates within a clinical context is to present risk of disease due to genetic and nongenetic factors side-by-side. This option, which our research team is currently pursuing, requires knowledge of an individual's medical, lifestyle, and family history. Granted for some diseases, risks due to lifestyle behaviors or other nongenetic factors that are relevant for all demographic groups are not available. However, presenting genetic risk alone and applying a threshold of the population average most often does not place reported risks within a meaningful clinical context.

Catharine B. Stack, PhD, MS

Coriell Personalized Medicine Collaborative Coriell Institute for Medical Research Camden, New Jersey

Disclosure: The author declares no conflict of interest.

\section{REFERENCES}

1. Mihaescu R, van Hoek M, Sijbrands EJ, et al. Evaluation of risk prediction updates from commercial genome-wide scans. Genet Med 2009;11:588-594.

2. van Hoek M, Dehghan A, Witteman JC, et al. Predicting type 2 diabetes based on polymorphisms from Genome-Wide Association Studies: a populationbased study. Diabetes 2008;57:3122-3128.

3. Wilson P, D'Agostino R, Levy D, Belanger AM, Silbershatz H, Kannel WB. Prediction of coronary heart disease using risk factor categories. Circulation 1998;97:1837-1847

4. Grundy SM, Cleeman JI, Mairey Merz CN, et al; National Heart, Lung, and Blood Institute; American College of Cardiology Foundation; American Heart Association. Implications of recent clinical trials for the National Cholesterol Education Program Adult Treatment Panel III Guidelines. Circulation 2004;110:227-239.

5. Narayan KM, Boyle JP, Thompson TJ, Gregg EW, Williamson DF. Effect of BMI on lifetime risk for diabetes in the U.S. Diabetes Care 2007;30:15621566 .

\section{Reply to "Disease risks derived from genetic variants need clinical context"}

\section{To the Editor:}

r. Stack correctly points out two limitations of genomewide scans used for predicting common diseases: the clinical context is lacking, and there is limited utility in comparing individual risks with the average risk. To evaluate the utility of tests, defining the clinical context is crucial. A genetic test usually is intended for a specific population and useful only if it changes health decisions, for example, if test results lead to different recommendations or different interventions. In some instances, testing can be beneficial also in the absence of interventions because people may value the information gained from learning about their health risks. This benefit is proven for monogenic diseases, such as Huntington disease, but unclear for complex diseases. ${ }^{1}$

The question arises what is the clinical context in predictive genetic testing for type 2 diabetes, which we used as an example in our analysis? ${ }^{2}$ Currently, there are no guidelines on risk thresholds for type 2 diabetes prevention, ${ }^{3}$ similar to the thresholds of the Framingham risk score for cardiovascular disease. ${ }^{4}$ Furthermore, the only available preventive strategy is adoption of a healthy lifestyle, which is recommended to all and should not need a genetic test to justify it. However, many companies promote that genetic tests will motivate preventive behavior. They argue that motivation increases when people learn that they are at higher risk than average. Whether their tests can 